Western University

Scholarship@Western

Winter 2-1-2016

\title{
Design and performance evaluation of a prototype MRF-based haptic interface for medical applications
}

\author{
Mehrdad Kermani Ph.D., P.Eng. \\ Western University, mkermani@eng.uwo.ca \\ Nima Najmaei \\ Engineering Services Inc.
}

Ali Asadian

$X($ Google X)

Rajni Patel

Western University, rvpatel@uwo.ca

Follow this and additional works at: https://ir.lib.uwo.ca/electricalpub

Part of the Biomedical Commons, Computer Engineering Commons, and the Controls and Control Theory Commons

\section{Citation of this paper:}

Kermani, Mehrdad Ph.D., P.Eng.; Najmaei, Nima; Asadian, Ali; and Patel, Rajni, "Design and performance evaluation of a prototype MRF-based haptic interface for medical applications" (2016). Electrical and Computer Engineering Publications. 190.

https://ir.lib.uwo.ca/electricalpub/190 


\begin{abstract}
This paper describes the construction and stability and transparency evaluation of a prototype two degrees-of-freedom (DoF) haptic interface, which takes advantage of MagnetoRheological Fluid (MRF) based clutches for actuation. These small-scale clutches were designed in our lab, and their evaluation were reported previously [1][2]. MRF-based actuators exhibit superior characteristics, which can significantly contribute to transparency and stability of haptic devices. Based on these actuators, a distributed antagonistic configuration is used to develop the 2-DoF haptic interface. This device is incorporated in a master-slave teleoperation setup intended for medical percutaneous interventions and soft-tissue palpation. Preliminary studies on the stability and transparency of the haptic interface in this setup using phantom and $e x$ vivo samples show the great potential of MRF-based actuators for integration in haptic devices that require reliable, safe, accurate, highly transparent, and stable force reflection.
\end{abstract}

\section{INTRODUCTION}

With the rapid growth in applications of minimally invasive medical interventions in recent years, the integration of haptics in such applications has been identified as a high-priority objective in major medical robotics roadmaps [3][4]. Motivated by in-depth research, it is not difficult to imagine the benefits of providing a clinician who is using robotics-based systems with the feel of being directly in contact with tissue and organs. Such sensation can improve the intuitiveness, safety, and efficiency of minimally invasive interventions [5]. However, the introduction of haptics in medical applications raises a number of safety concerns [6][7]. The issue of stability of haptics-enabled telerobotic systems [8] is a major obstacle in introducing haptics into medical environments [4]. As a result, the application of haptics in medicine is almost nonexistent and is highly limited to research. Thus, much research has focused on developing reliable control techniques for haptic applications. To this end, the use of passivity-based and smallgain approaches has been reported [9][10]. However, the conservative nature of such methods results in degraded quality of force reflection, which decreases the efficiency of these devices. Another body of work has focused on addressing this

The authors are with the Dept. of Electrical and Computer Engineering, Western University, London ON., Canada, as well as, with Canadian Surgical Technologies \& Advanced Robotics (CSTAR), London ON, Canada. R.V. Patel is also with the Dept. of Surgery, Western University. Financial support was provided by an NSERC CGS-D Scholarship (N. Najmaei), an Ontario Graduate Scholarship (N. Najmaei), an NSERC Collaborative Research and Training Experience (CREATE) program grant (R.V. Patel) on ComputerAssisted Medical Interventions, and NSERC grants RGPIN-1345 (R.V. Patel) and RGPIN-346166 (M.R. Kermani). A. Asadian was supported by a postdoctoral fellowship from the NSERC CREATE program on Computer-Assisted Medical Interventions (R.V. Patel). The design of the haptic interface described in this paper is part of a project on haptic devices for medical interventions and training (led by R.V. Patel) that is funded by an Ontario Research Fund Research Excellence Grant (RE-05-049).

Corresponding Author: Nima Najmaei,nnajmaei@uwo.ca issue through redesigning the actuation mechanisms in haptic interfaces. The actuators and mechanisms used in designing a haptic interface play an important role on the quality of force feedback, as well as the stability of the system. In order to have transparency, it is of utmost importance that while a haptic device is capable of recreating the forces that occur during contact with stiff objects, it exhibits low friction, damping, and inertia to sustain transparency during motion in free space. Poor dynamics and control of manipulation (e.g., backlash, unmodeled nonlinear dynamics, etc.) can affect the sense of touch, in particular when rigid instruments or actuators are used [11]. The use of electrical motors in haptic devices, especially for medical applications, has proved to be challenging for several reasons. The poor dynamics of electrical motors, imposed by the need for gear reduction, can significantly reduce the transparency of the system by increasing the damping, inertia, and friction of the haptic device [12]. This is highly undesirable in medical applications that require high-fidelity and accurate force reflection. Moreover, their active nature (which may be exhibited in the form of oscillations and jerks) can result in degradation of stability, which is unacceptable in medical applications [8][13].

In recent years, actuators based on Magneto-Rheological fluids have been proposed as an alternative for use in haptic devices [11]. MRFs exhibit a very unique characteristic: the viscosity and shear stress of these fluids can be intelligently changed using an applied magnetic field. The electromechanical characteristics of MRF-based actuators make them a great candidate for use in haptic devices. MRFs are capable of producing high shear stress, which enables the design of actuators that are capable of generating high force and torque, without the need for gear reduction [12][14]. Moreover, MRF-based devices can be made substantially compact and lightweight, and they exhibit low inertia- and mass-torque ratios [12]. All the aforementioned factors play an important role in the transparency of a haptic interface. Furthermore, devices such as MRF-based clutches, are capable of creating a controllable damping and matching large impedance contacts [15] which contributes to stability of the system as is known from passivity and small-gain theorems [10]. This claim was experimentally validated in our previous work [16]. These characteristics have motivated the design of several haptic devices based on Magneto-Rheological and ElectroRheological Fluids (ERF), which include, but are not limited to, (i) joysticks [17][18], (ii) force displays [13][19][20], and (iii) hand masters [21][22][23][24]. Table I summarizes the size and torque capacity of some of the actuators used in these devices. Due to the poor torque-to-volume ratio of their actuation system, none of these studies were capable of achieving the size and strength offered by commercial haptic 
devices. As a result, in our previous work [1], a new design, namely Armature-Based MRF clutch, was proposed which has superior torque-to-volume ratio in comparison with the conventional designs (Table I). Four prototype clutches were constructed based on this design and were evaluated in [2]. The results show the great potential of these actuators to be used in haptic devices with improved stability and transparency. This paper reports on the design and development of a prototype two-DoF haptic interface based on the developed MRF-based clutches. This device is used to evaluate the stability and transparency of such clutches in bilateral teleoperation systems, and compare that to those of commercial hepatic interfaces. The results demonstrate the great benefits that a MRF-based actuation system brings to a teleoperation system and show the significant potential of MRF-based haptic devices for use in medical applications that require reliable, accurate, and stable force reflection. This is the main contribution of this paper. To this end, in Section II, a distributed antagonistic configuration is introduced which enables the construction of a 2-DoF haptic interface based on the constructed actuators [2]. Section III provides a brief discussion on the stability and transparency of a haptic interface as the performance measure of such devices. The specifications of the developed system are compared with three well-known off-the-shelf haptic interfaces and the contribution of this work to the state of the art is discussed in Section IV. This device is then incorporated in a master-slave teleoperation setup that is used for percutaneous and soft-tissue applications in Section V. The virtual wall benchmark is used in Section VI-A to assess the stability of teleoperation using the MRF-based haptic device, which shows great improvement over the PHANTOM Omni and Quanser Haptic Wand. Next, several experiments are performed using phantom and ex vivo samples to further study the stability and transparency of the system in Sections VI-A and VI-B.

\section{Design And Control of the Two-DoF Haptic INTERFACE}

This section describes the mechanism used to design a two-DoF MRF-based haptic interface. First, a review on MRF-based clutches [1] is given. Next, the distributed antagonistic actuation mechanism that is required to construct a multi-DoF haptic interface based on these actuators is discussed.

TABLE I

COMPARISON OF MRF-BASED ACTUATORS USED IN HAPTIC INTERFACES $(\varnothing$ DENOTES THE DIAMETER SIZE)

\begin{tabular}{lccc}
\hline Research & Type & Dimensions & Torque \\
\hline \hline Najmaei et al. [2] & Armature & $\varnothing 3.6 \times 6 \mathrm{~cm}$ & $1.3 \mathrm{Nm}$ \\
Melli-Huber et al. [17] & Disk & $\varnothing 4.3 \times 2 \mathrm{~cm}$ & $0.7 \mathrm{Nm}$ \\
Furusho et al [19] & Disk & $\varnothing 20 \mathrm{~cm}$ & $2.0 \mathrm{Nm}$ \\
Yamaguchi et al. [20] & Disk & $\varnothing 17 \mathrm{~cm}$ & $10 \mathrm{Nm}$ \\
Blake et al [24] & Disk & $\varnothing 2.5 \times 1.4 \mathrm{~cm}$ & $0.2 \mathrm{Nm}$ \\
Liu et al. [25] & Disk & $\varnothing 15 \mathrm{~cm}$ & $0.9 \mathrm{Nm}$ \\
Nguyen et al. [26] & Disk & $\varnothing 10 \mathrm{~cm}$ & $10 \mathrm{Nm}$ \\
Ahmadkanlou et al. [14] & Drum & $\varnothing 8 \times 10 \mathrm{~cm}$ & $1.5 \mathrm{Nm}$ \\
\hline
\end{tabular}

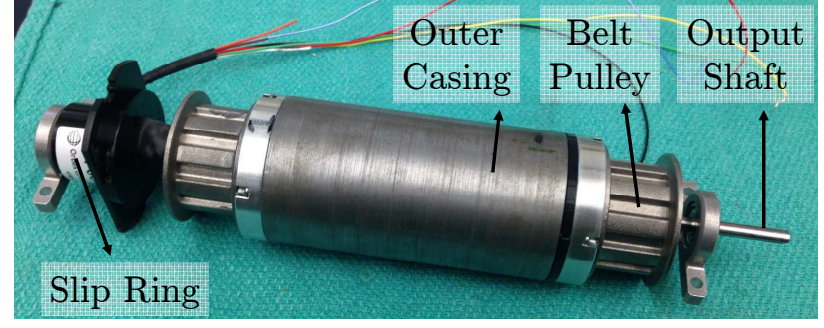

Fig. 1. The MRF-based clutch used for actuation of the haptic interface.

MRF-Based Actuators: Fig. 1 shows one of the constructed clutches. The structure of these clutches is as follows: The MRF fills the gap between a cylindrical outer casing and an armature, made of ferromagnetic materials. The armature is equipped with magnetic coils (similar to a DC Motor), which can create an axial magnetic field inside the MRF gap. The outer casing and armature are decoupled through the use of ball bearings and both can rotate freely. The caps of the outer casing are sealed to contain the MRF inside the gap. A clutch is responsible of controlling the amount of torque transferred from the input (outer casing) to the output (armature), similar to an automobile's clutch. To this end, a driving motor applies a constant input velocity to the outer casing of the clutch (input). By controlling the magnetic field, the viscosity and shear stress of MRF inside the gap are changed, and subsequently the share of the input torque that is transferred to the output shaft can be controlled. Hall sensors are embedded in the system to measure the magnetic field strength. Belt pulleys are mounted on the outer casing cap to apply the input torque to the clutch. The output torque is delivered to the output link using cables and capstan pulleys. For more information please refer to [1][2]. The length and diameter of each clutch are $60 \mathrm{~mm}$ and $36 \mathrm{~mm}$, respectively; weight is 598gr, the output inertia is $427 \mathrm{gr.cm}{ }^{2}$, and each has the capability of generating $1.5 \mathrm{Nm}$ of torque. The bandwidth of these actuators is $30 \mathrm{~Hz}$.

Bidirectional Actuation: Note that, an MRF-clutch on its own is only capable of generating a passive/resistive torque. Hence, as mentioned, the combination of a clutch and an active motor should be used to create active torque and motion (which is called semi-active actuation). However in this formation, the clutch is only capable of providing motion in the same direction as the motor. The trivial solution to this issue is to control the direction of the motion by changing the direction of the motor. However, this method adds to the size, weight, and mechanical complexity of the device (each clutch needs one driving motor). To avoid this problem and to achieve bidirectional actuation, an antagonistic configuration is used. In this configuration, clutches work in pairs to provide motions in both directions. A single driver motor applies the input torque to all clutches in the same direction. However, the cable attaching the capstan pulleys is installed in an $\infty$ shape (see cable connections in Fig. 3). In this way, the output torques of the clutches are applied in opposite directions, which enables bidirectional actuation of the link through a push-pull cable mechanism. Hence, by switching between the two clutches, the direction of operation can be changed, while 
the direction of the motor remains the same [27][28][29][30]. The antagonistic configuration provides a major benefit for the haptic device. Any friction in the system can degrade the transparency of the system. Different factors can contribute to friction in the system. The viscosity of the fluid creates an off-state torque. Any residual magnetic field in the system can result in a biased shear stress and the user will feel added friction as a result. Also the seals that are used to avoid leakage of the fluid can create large friction. Although this could be a serious concern, the antagonistic configuration presented here addresses this problem to a great extent. This is due to the fact that the created friction forces in a pair of clutches antagonistically connected to a single joint will have similar magnitude, but in opposite directions [29]. Hence, the friction forces will tend to balance each other out. This is a useful property for haptic devices, which require minimal friction for high transparency.

Expansion to Multi-DOF: Furthermore, the use of MRF-based clutches in a multi-DoF haptic device can be addressed using the concept of Pluralized Antagonistic Distributed Active Semi-Active (PA-DASA) actuation [27]. This is an expansion of the antagonistic configuration described previously as shown in Fig. 2. In this structure multiple MRF clutches can be driven by a single motor (active actuator), which is located at the base of the haptic interface to keep the link masses low. The single motor applies a constant unidirectional velocity to all sets of clutches through a system of cables and pulleys. A set of two clutches is employed in an antagonistic configuration to apply torques to each joint through cables and pulleys, independently [27]. The torque direction and magnitude at each joint can then be changed by controlling the magnetic field in the corresponding MRF clutch pair, without altering motor direction or torque. This has the benefit of rectifying a possible dead-zone due to motor direction change. This concept is the key strategy behind developing the multi-DoF haptic interface. The PA-DASA mechanism is intended to increase the performance of the interface, while offering reduced mass and effective inertia at all joints over using conventional servo motors [28][31]. It should be noted that the only control required at the single motor stage is to provide constant velocity. The application of PA-DASA in large-scale two- and three-DoF industrial robots has been assessed in our group and the results have shown the suitability of such a configuration [28][29]. Hence, by taking advantage of the small-scale MRF-based clutches in a PA-DASA configuration, a lightweight and compact multi-DoF haptic interface can be made. The structure of a two-DoF haptic interface constructed based on the aforementioned principles is shown in Figs. 3 and 4 . A brushless Maxon EC-60 DC motor is used to drive four clutches. A 3-DoF planar haptic handle is also constructed using rapid prototyping. A system of cables and capstan pulleys is used to transmit bidirectional torques from each pair of clutches to the joints (2-DoF). A third passive joint is also incorporated. Table II shows the specifications of this device.

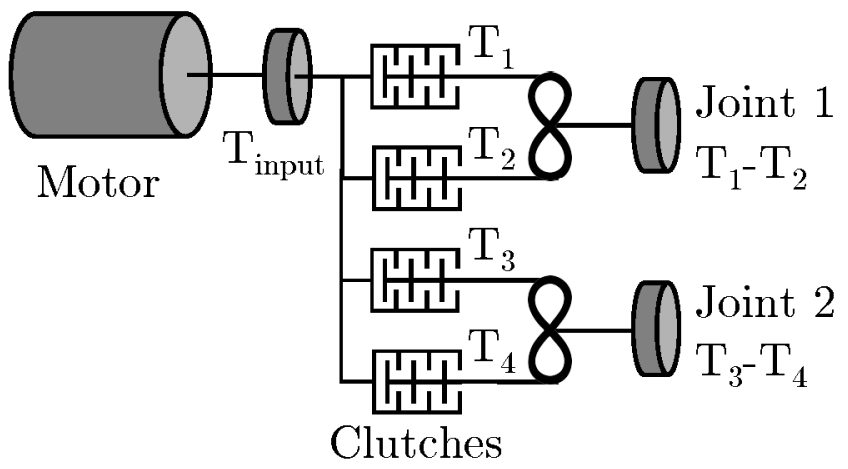

Fig. 2. Schematic view of PA-DASA Configuration. The input torque from the motor $\left(\mathrm{T}_{\text {input }}\right)$ is applied to all four clutches. Each clutch individually controls the amount of torque transferred to its output $\left(\mathbf{T}_{1}\right.$ to $\left.\mathbf{T}_{4}\right)$, and each pair of antagonistic clutches controls the bidirectional output torque applied to each joint $\left(\mathrm{T}_{1}-\mathrm{T}_{2}\right.$ for joint 1 and $\mathrm{T}_{3}-\mathrm{T}_{4}$ for joint 2$)$.

Modeling and Control: Reliable control and actuation is a critical issue in haptic devices for delivering accurate and high-fidelity feedback, especially in case of medical applications. MRF-based actuators suffer from nonlinear hysteretic relationships between the input current and magnetic field, as well, as the magnetic field and shear stress (output torque). This nonlinearity can cause inaccuracy in the output response of the actuator, as well as, instability in the closed-loop system. Therefore, it is essential to study and model the current-torque relationship for MRF-based actuators [32]. A modeling and control scheme for such actuators has been proposed in [2]. In this section, this method is briefly discussed; A simple PID controller provides the control current for a desired torque value to the coil of the clutch. This controller uses the error between an estimated value of the output torque with its desired value as the input signal. The main goal of this control technique is to avoid using any external force/torque sensor [33]. To this end, an approximator based on an artificial neural network (ANN) is used to predict and model the output torque of the system (which is used for feedback), just based on the measurement of the magnetic field strength by the embedded Hall sensors in the clutch. The ANN is trained based on a set of pre-recorded measurements. The results in [2] showed the efficient performance of this modeling and control strategy.

REMARK 1. The design of the haptic interface needs further modifications based on optimization to achieve desired characteristics for a proper haptic device. However, at this stage, our main focus is on evaluating the performance of MRF-based actuation mechanisms for haptic applications and this device enables us to achieve this goal.

TABLE II

HAPTIC INTERFACE SPECIFICATIONS

\begin{tabular}{lc}
\hline Parameter & Value \\
\hline \hline Haptic Box Dimensions $[\mathrm{mm}]$ & $200 \times 170 \times 100$ \\
Length of Links 1, 2, $3[\mathrm{~mm}]$ & $100,80,50$ \\
Joints Motion Range 1, 2[deg] & 200,190 \\
Maximum Joint Torque $[\mathrm{Nm}]$ & 1.5 \\
Maximum Cartesian Force $[\mathrm{N}]$ & 10 \\
\hline
\end{tabular}




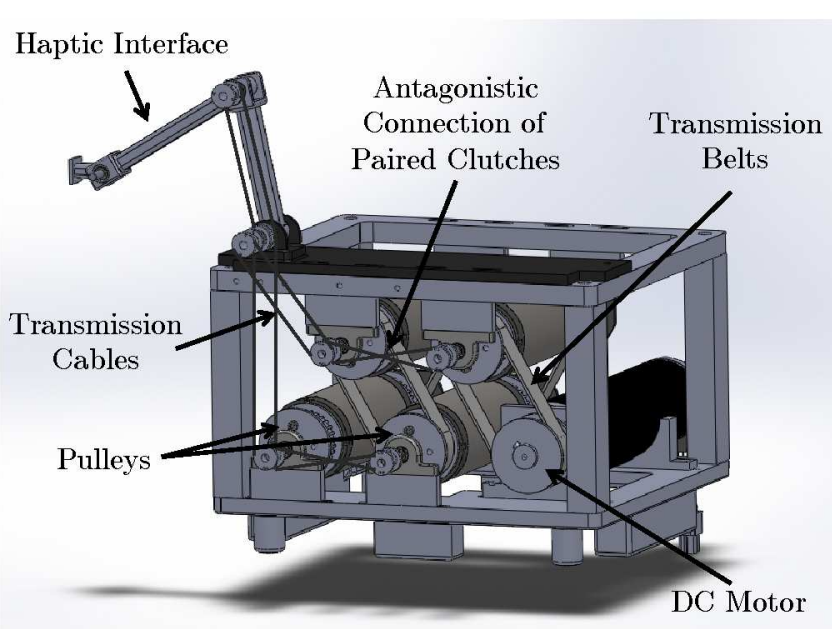

Fig. 3. PA-DASA structure of the multi-DoF haptic interface.

\section{Performance Measures of Haptic Devices}

A discussion on stability and transparency as the main measures for evaluating the performance of haptic devices is given. This section serves as a preamble for the subsequent sections in which these factors are experimentally evaluated and compared with corresponding factors for commercial haptic devices.

\section{A. Stability and Z-Width}

An important measure of a haptic interface performance is the dynamic range of achievable impedance while a property such as passivity or stability is satisfied [34]. This range is called Z-Width. A well-known benchmark for studying the stability and the Z-Width of a system is the virtual wall experiment. A virtual wall can generate energy which subsequently may result in the system becoming unstable [35]. Using the passivity theorem [36], a necessary and sufficient condition for the stability of a haptic interface with physical damping of $b_{p}$ interacting with a virtual wall with virtual stiffness $K_{W}$, virtual damping $B_{W}$, and sampling time $T_{s}$, can be derived. Such a condition, which shows the Z-Width of the system, implies the maximum achievable virtual stiffness and virtual damping [37] and is given by,

$$
b_{p}>\frac{K_{W} T_{s}}{2}+B_{W}
$$

In the following sections, the improvement of Z-Width in MRF-based haptic interfaces is experimentally studied.

\section{B. Transparency}

The transparency of a haptic interface has two folds. On one hand, it is directly affected by the dynamics of the actuation mechanism (e.g., inertia- and mass-torque ratios). It was shown in our previous papers [1][2], that MRF-based actuators have better dynamical characteristics in comparison to electrical motors, which significantly contribute to the transparency of the system. On the other hand, the issues of transparency and stability are intertwined. To better describe this relationship, this paper takes advantage of the small-gain theorem in this

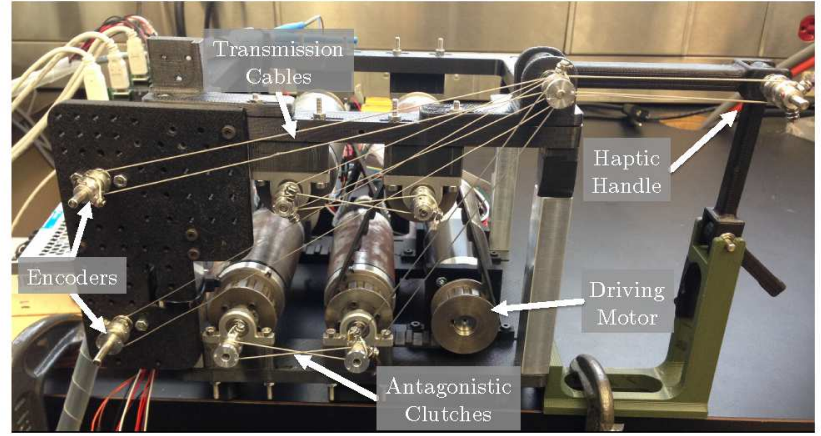

Fig. 4. The 2-DoF haptic interface.

section. The small-gain theorem, which is an important result in control system theory, indicates that a feedback system is stable if the product of the IOS (Input-Output Stability) gains [38] of its subsystems is less than one [39]. This result is applicable to a wide range of dynamical systems whose parts communicate over networks. In a teleoperation system, a master device, with impedance $Z_{m}$, is interconnected to a slave device and environment, with combined impedance of $Z_{s e}$, through a communication channel. A filter with the variable gain of $\gamma_{f}$ can be used to guarantee the stability of the system by attenuating the magnitude of the force reflected back to the user [40][41]. To have good transparency, it is desirable to have a $\gamma_{f}$ close to 1 . By considering some assumptions, the condition of stability for this system is obtained as [40][41],

$$
\gamma_{f}<\frac{\left\|Z_{m}(s)\right\|_{\mathcal{L}_{1}}}{\left\|Z_{s e}(s)\right\|_{\mathcal{L}_{1}}}
$$

It is evident from this equation that if a haptic interface has a large Z-Width, the transparency of the system holds $\left(\gamma_{f}\right.$ close to 1) for a larger range of environment impedances. In other words, if the master device is not capable of generating impedances as high as the environment, then the transparency will need to be sacrificed $\left(\gamma_{f} \ll 1\right)$ to achieve stability. In the following sections, the improvement of transparency in MRFbased haptic interfaces is experimentally studied by examining the Z-Width and accuracy of force feedback.

\section{Comparison with Commercial Haptic Devices}

In this section, specifications of the prototype MRF-based haptic device are compared with those of three well-known off-the-shelf haptic interfaces, namely Geomatic PHANTOM Omni [42], Geomatic PHANTOM Desktop [42], and Quanser Haptic Wand [43]. Based on the findings of [44], several performance measures were considered to compare these devices. These factors are listed in Table III. It is evident that, while the developed device has fewer degrees of freedom and comparable weight and dimensions to the other devices, it is capable of producing the largest force, torque, and stiffness. As mentioned in [2], the weight and size of the device can be reduced in the next generation of the system through using more appropriate machining techniques. About $1 \mathrm{~kg}$ of the device weight is the weight of the Maxon EC-60 motor used in the setup for driving the clutches. This motor can be replaced with a lighter and less powerful one, since the PADASA configuration reduces the need for a powerful servo 
TABLE III

COMPARISON TO OFF-THE-SHELF HAPTIC INTERFACES

\begin{tabular}{lcccc}
\hline \hline Parameter & $\begin{array}{c}\text { Prototype } \\
\text { Device }\end{array}$ & $\begin{array}{c}\text { PHANTOM } \\
\text { Omni }\end{array}$ & $\begin{array}{c}\text { PHANTOM } \\
\text { Desktop }\end{array}$ & $\begin{array}{c}\text { Quanser } \\
\text { Wand }\end{array}$ \\
\hline DoF (w/ Feedback) & 2 & 3 & 3 & 5 \\
Length [mm] & 200 & 184 & 203 & 450 \\
Width [mm] & 170 & 143 & 168 & 335 \\
Height [mm] & 100 & 70 & 120 & 350 \\
Weight [kg] & 3.5 & 1.8 & 3.1 & 20.0 \\
Off-state Force [N] & 0.2 & 0.06 & 0.26 & - \\
Cont. Force [N] & 8.0 & 0.9 & 1.8 & 3.0 \\
Max. Force [N] & 14.0 & 3.3 & 7.9 & 9.0 \\
Max. Joint T [Nm] & 1.5 & - & - & 0.96 \\
Stiffness [kN/m] & 18 & 2.3 & 2.35 & 6 \\
\hline \hline
\end{tabular}

system. Nevertheless, the developed device, as constructed, demonstrates the great potential of MRF-based actuation (in terms of size, weight, and torque capacity) for use in haptic interfaces.

\section{EXPERIMENTAL SETUPS}

Fig. 4 shows the developed 2-DoF prototype haptic device. In this section, the experimental setups used for validating the performance of the developed MRF-based haptic interface are introduced. The haptic interface operates as follows; each joint of the haptic handle is connected through cables to an antagonistic pair of clutches. A switching algorithm activates the desired clutches based on the direction of the feedback force. Four PID controllers (implemented in software) are used to control the output torque of the clutches at $1 \mathrm{KHz}$. The gains of this controller were empirically obtained and set to 2.35 , $0.4,0.03$ for the proportional, integral, and derivative gains, respectively. As mentioned in before, the ANN model is employed to provide the feedback signal of the PID controllers, based on the readings of the embedded Hall sensors. The coils of clutches are connected to individual current drivers (Maxon EPOS 50/5 controller operating in current mode), which controls the current of the coil at $1 \mathrm{KHz}$. The PID controller provides the set-point for the current drivers. The joints angles of the haptic handle are measured using two optical encoders (with 1024 counts per revolution). A data acquisition card (Sensoray 626) is employed to provide the current drivers with the reference current (from PID controller), as well as, to read the output of the Hall sensors and encoders. The driving motor (Maxon EC-60) is also controlled using a Maxon EPOS 50/5 controller, operating in constant velocity mode. It what follows the configurations used to evaluate the performance of the haptic device are reviewed.

\section{A. Configuration A: Virtual Wall Experiments}

To evaluate the stability of the system, a virtual wall experiment was implemented. A virtual wall consisting of a linear spring with stiffness $K_{W}$ and a damping $B_{W}$, was implemented on a computer (different from the one used for controlling the haptic interface). The wall is in the form of a plane at $x=0$ in the Cartesian space. The two computers communicate through a UDP connection (Configuration $A$ ). The location of the haptic handle end effector is sent to the slave computer. The virtual wall applies force when the end effector passes the virtual wall (i.e., $x<0$ ). The reflected force information is sent to the master side and the haptic handle applies force to the operator's hand. To compare the performance of the prototype device with a Geomatic PHANTOM Omni and a Quanser Haptic Wand, these devices were used in place of the MRF-based interface in the same configuration.

\section{B. Configuration B: Needle Insertion Experiments}

The performance of the haptic interface is also evaluated in a needle insertion and steering application from medical robotics. The large variations in forces experienced when a needle punctures into tissue provides us with the proper challenge for testing the designed system. Fig. 5 presents the block diagram of the master-slave haptic teleoperation system used for medical needle insertion (Configuration $B$ ).

In our experimental setup, a state-of-the-art robotic system designed in our group at CSTAR for percutaneous needle insertion [45] was used as the slave robot. This 5-DoF manipulator can perform orientation, insertion, and rotation of the needle and linear motion of the stylet to drop radioactive seeds in the prostate during the brachytherapy procedure. To test the haptic device, only the $1-\mathrm{DoF}$-insertion force was fedback to the user. A 6-DoF ATI Nano43 force/torque sensor was mounted at the base of the needle holder combined with the force estimation technique developed in [46] was used to provide force reflection. PID controllers have been implemented to control the position of the needle at $1 \mathrm{KHz}$. The needle used in this setup is an 18GA (Cook Medical), with a conical tip. The master and slave sides communicate with each other through internet using UDP protocol. Figs. 6(a) show the needle insertion robot and the needle insertion mechanism in Configuration $B$.

\section{Configuration C: Tissue Palpation Experiments}

The performance of the haptic interface is also evaluated using a tissue palpation application (Fig. 6(b)). The large forces experienced during palpation (e.g., for tumor detection), will provide us with the proper challenge for evaluating the designed system. This configuration is basically the same

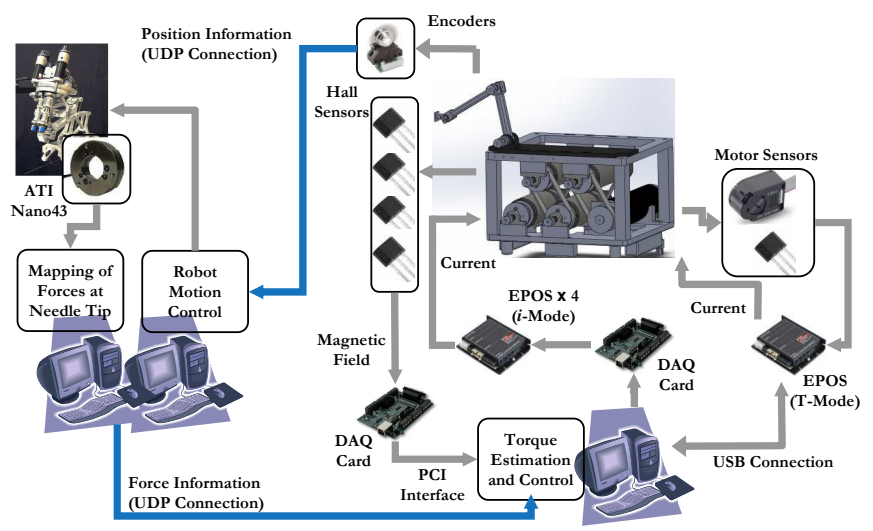

Fig. 5. Configuration B: Block diagram of the master-slave haptic teleoperation. 


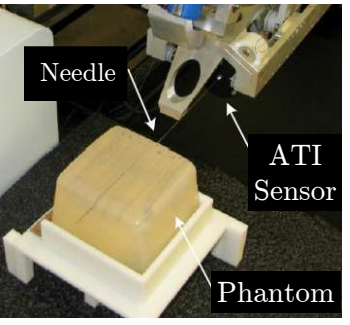

(a)

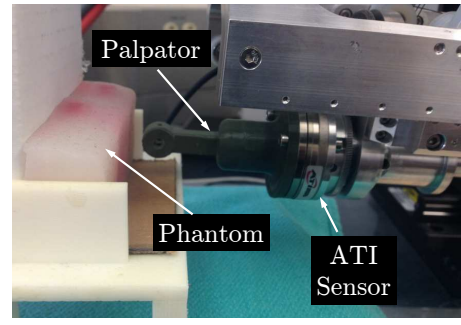

(b)
Fig. 6. (a) Configuration $B$ : Needle insertion setup, (b) Configuration $C$ : Tissue palpation setup.

as Configuration $B$; however, the needle is replaced with a palpator tool, which was made by rapid prototyping.

\section{Configurations $B^{*}$ and $C^{*}$ : Force Feedback Transparency}

To evaluate the accuracy of the device in providing accurate force feedback, Configurations $B^{*}$ and $C^{*}$ were considered. These are similar to Configurations $B$ and $C$. However, a customized third link was constructed for the handle, which allowed the end effector to be attached to an ATI Nano43 sensor. This force sensor was mounted on a linear stage, as shown in Fig. 7. The linear stage was used to move the handle. The sensor measures the feedback force and provides a means for evaluating the accuracy of force feedback.

\section{E. Configurations: Summary}

A summary of the configurations used in the experiments of this paper is given in Table IV. Note that, for the purpose of comparison, the PHANTOM Omni or Quanser Haptic Wand can be replaced with the MRF device in any of these configuration.

\section{RESULTS}

\section{A. Evaluation of Force Feedback Stability}

In this section, the stability of the developed MRF-based haptic interface is compared with that of a PHANTOM Omni

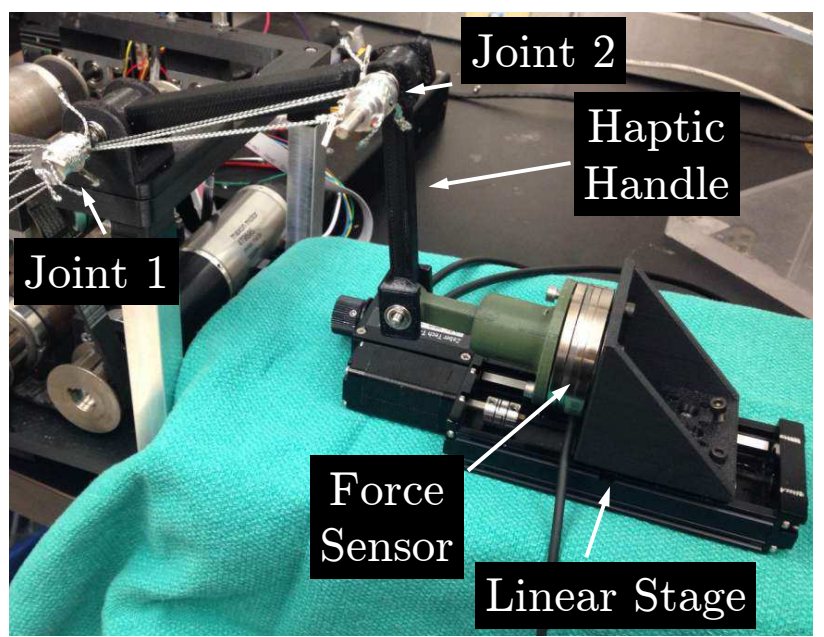

Fig. 7. Configuration $B^{*}$ and $C^{*}$ : Use of a linear stage and a sensor for validating haptic feedback transparency using a large variety of samples.
TABLE IV

SUMMARY OF CONFIGURATIONS USED IN THE EXPERIMENTS

\begin{tabular}{lll}
\hline \hline Config. & Structure & Validation Goal \\
\hline Conf. A & MRF Device + Virtual Wall & Stability \\
Conf. B & MRF Device + Needle Insertion Setup & Stability \\
Conf. $B^{*}$ & Conf. B + Linear Stage + ATI Nano 43 & Transparency \\
Conf. C & MRF Device + Palpation Setup & Stability \\
Conf. $C^{*}$ & Conf. C + Linear Stage+ ATI Nano 43 & Transparency \\
\hline
\end{tabular}

device and a Quanser Haptic Wand through several tests. To this end, first, the virtual wall benchmark is implemented, as described in Configuration A, and all devices are tested in different scenarios. Next, phantom and ex vivo samples are used in Configurations $B$ and $C$ to compare the developed system's stability in real-world applications.

1) Virtual Wall Experiments: As mentioned, a virtual wall was implemented in Configuration A. Two sets of experiments were conducted; (1) For different values of virtual damping $B_{W}$, the maximum achievable virtual stiffness $K_{W}$ by the MRF-based device, the PHANTOM Omni, and the Quanser Haptic Wand was obtained and compared, i.e., by studying the Z-Width, as described in [1]. (2) Robustness of the stability of these devices to the variation of sampling time were evaluated and compared.

a) Study of Z-Width: In these experiments, Configuration A was used to study the $Z$-Width of the system. To this end, for different values of virtual damping the maximum achievable virtual stiffness, for a stable operation of MRF-based haptic device, PHANTOM Omni, and Haptic Wand, was obtained. All the experiments in this section were performed at $1 \mathrm{KHz}$. Fig. 8(a) shows the results. As can be seen, the MRF-based haptic interface exhibits the largest Z-Width in comparison with the other two haptic devices. These results once more support the conclusion drawn in [1] and show the significant contribution of these type of actuators in improving the stability of a teleoperation system. Fig. 9 presents the results of some of these experiments. In these tests, a virtual damping of $B_{W}=6 \frac{N . s}{m}$ was considered. Note that the virtual slave comes into contact for positions less than or equal zero. Figs. 9(a-c) present the stable interaction of the Omni, Haptic Wand, and MRF-based devices with a wall with stiffness of $K_{W}=500,2000$, and $10000 \frac{N}{m}$, respectively. However, Figs. 9(d-f) show the result of haptic rendering of a virtual

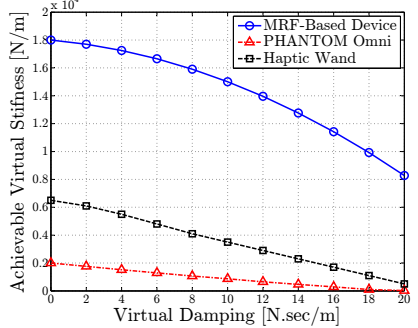

(a)

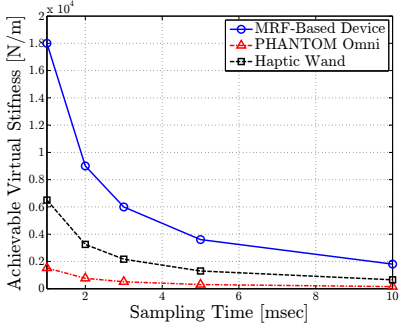

(b)
Fig. 8. Maximum virtual stiffness that the MRF-based device, PHANTOM Omni, and Haptic Wand can render while remaining stable for (a) variable virtual damping, and (b) variable sampling time (Configuration A). 


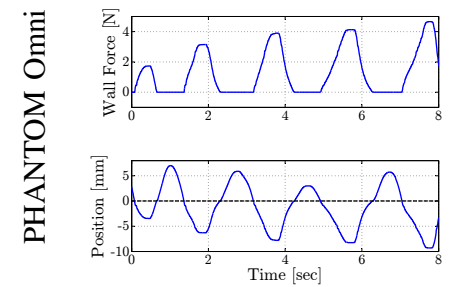

(a) $K_{W}=500 \mathrm{~N} / \mathrm{m}$

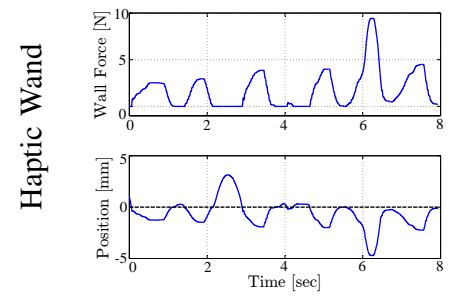

(b) $K_{W}=2000 \mathrm{~N} / \mathrm{m}$

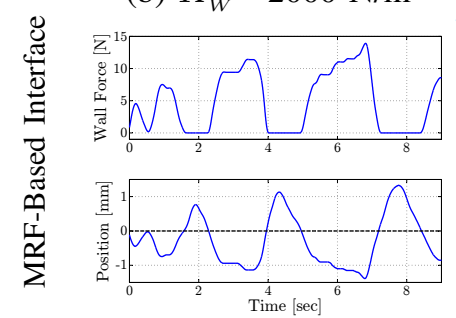

(c) $K_{W}=10000 \mathrm{~N} / \mathrm{m}$

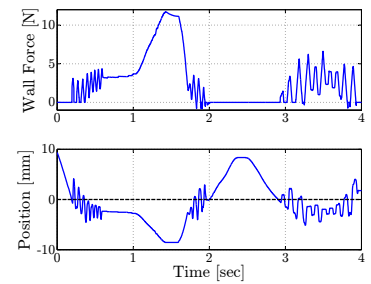

(d) $K_{W}=1300 \mathrm{~N} / \mathrm{m}$

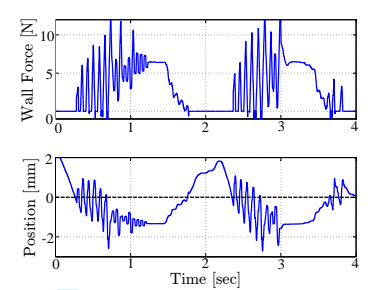

(e) $K_{W}=4800 \mathrm{~N} / \mathrm{m}$

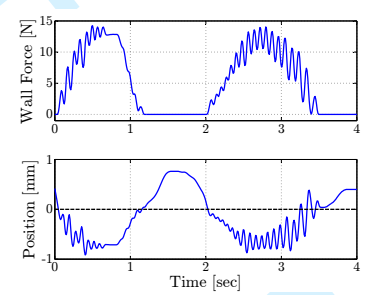

(f) $K_{W}=18000 \mathrm{~N} / \mathrm{m}$
Fig. 9. Results of interaction with the virtual wall (Configuration $A)$ at $1 \mathrm{KHz}$ with damping $B_{W}=6 \frac{\mathrm{Ns}}{\mathrm{m}}$ and different stiffness for PHANTOM Omni, Haptic Wand, and the prototype system. (a-c) Stable interaction and (d-f) unstable interaction.

wall with stiffness just above the achievable stiffness. As seen, the MRF-based device remains stable for a very large range of virtual stiffness, which shows its high Z-Width.

b) Effect of Sampling Time on Stability: Next, the robustness of the system to changes in the sampling time, $T_{s}$, of the virtual wall is examined and compared with that for the Omni and the Haptic Wand. For this purpose, a constant virtual damping $\left(B_{W}=4 \frac{N . s}{m}\right)$ was considered. The sampling time of the virtual wall was varied and once more the maximum achievable virtual stiffness was obtained. Note that the haptic interface still provides feedback at $1 \mathrm{KHz}$. Fig. 8(b) shows the results. Once more, these experiments proved that the MRF-based haptic device has larger stability margin and is more robust to the delay caused by the sampling time variation.

\section{2) Phantom and Ex Vivo Needle Insertion and Palpation:} In this section, phantom and ex vivo samples are used in needle insertion and tissue palpation experiments to evaluate the stability of the system in real-world applications.

REMARK 2. Phantom samples are used in these experiments to test the device for a wider range of stiffnesses. The elastic moduli of soft mammalian tissues range from near $100 \mathrm{~Pa}$ for the softest organs such as the brain, to tens of thousands of $\mathrm{Pa}$ in muscle tissues, and on the order of MPa in cartilage [47]. According to the measurements in [48][49], a 4\% gelatin phantom has similar stiffness to that of lung tissue in Guinea pig or a breast tumor in human. Based on the findings of [47][50], agar samples with concentration rates of $3 \%$, $6 \%$, and $10 \%$ have stiffness close to beef liver, muscle, and cartilage, respectively.

a) Needle Insertion: Configuration B was used in this section. A phantom made of agar with a concentration of $10 \%$ was used in these experiments. The user guided the needle inside the phantom using the haptic handle. Figs. 10(a-c) present the result of such interaction using the three considered haptic interfaces. During operation, the PHANTOM Omni became unstable and exhibited strong oscillations as can be seen in the plot. The Haptic Wand performed relatively better, however the ripples in the created force are highly undesirable for medical applications. As seen, the MRF-based haptic interface provided the most stable and desirable haptic feedback to the user. The user was able to make a very smooth insertion and retraction of the needle. In another set of experiments, the same test was performed on an ex vivo kidney sample (beef). This organ consists one of the stiffest tissues in the body due to its thick renal cortex. Figs. 10(d-f) show the results. Once more both the Omni and the Haptic Wand exhibit strong ripples and oscillations. On the other hand, the MRF-based haptic interface provided very stable interaction.

b) Tissue Palpation: In order to further study the performance of the developed haptic interface in medical applications, Configuration $C$ was used and several experiments on tissue

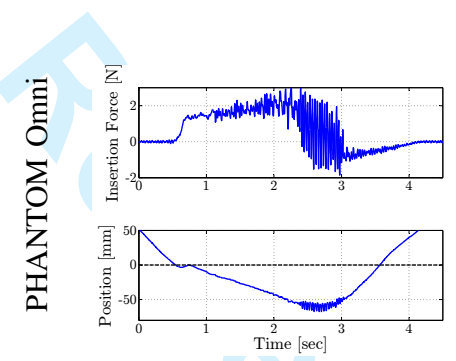

(a)

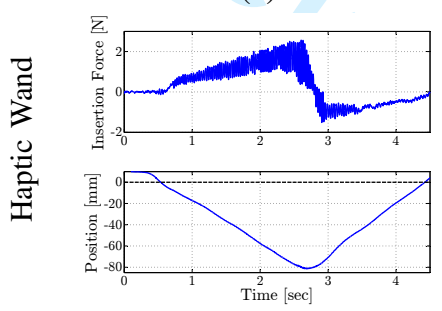

(b)

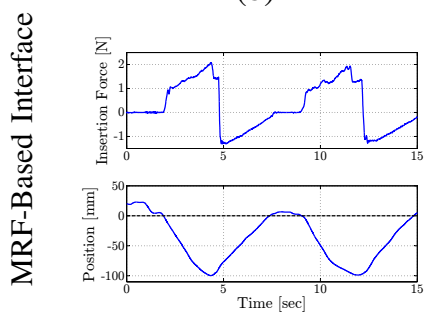

(c)

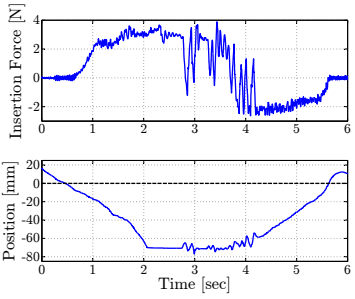

(d)

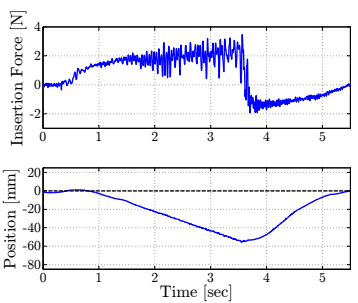

(e)

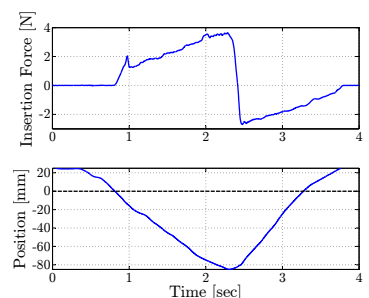

(f)
Fig. 10. Results of needle insertion and retraction (Configuration $B$ ) on (a-c) an agar sample ( $10 \%$ concentration) and (d-f) an ex vivo beef kidney. 
palpation were performed. This section shows the results of the palpation of a phantom made out of agar (10\%) and of an ex vivo kidney (beef). The user tried to make several contacts with the samples using the haptic device. The results are shown in Fig. 11. Once more, the significant improvement in stability of teleoperation through the use of MRF-based system is visible. The other devices become unstable as the contact is made, which is exhibited as uncontrolled motions of the handle. In case of MRF-based system, the interaction remains very stable. Note that the effectiveness of the MRF-based haptic interface in distinguishing between high stiffness tissues (tumor) and lower ones is showed in next section.

\section{B. Evaluation of Force Feedback Transparency}

The stability of the system was studied in the previous section and was compared with that of two other off-the-shelf haptic interfaces. In this section, the accuracy of force feedback provided by the MRF-based haptic interface is analyzed by taking advantage of Configurations $B^{*}$ and $C^{*}$. These tests were designed for (1) validating the accuracy and transparency of the feedback and (2) studying the ability of the haptic device to provide a differentiable feel to the user of different tissues with diverse stiffnesses.

REMARK 3. As discussed in Section III, the transparency of a haptic device depends on dynamical characteristics and control, as well as on the Z-Width of the system. In the previous section, the improvement in Z-Width over other

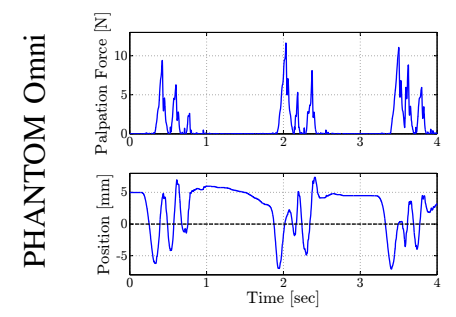

(a)

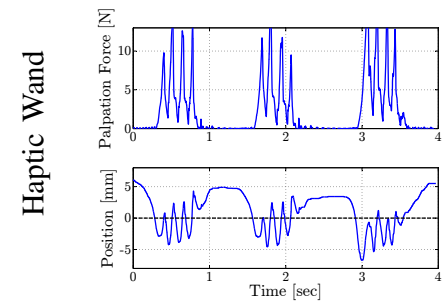

(b)

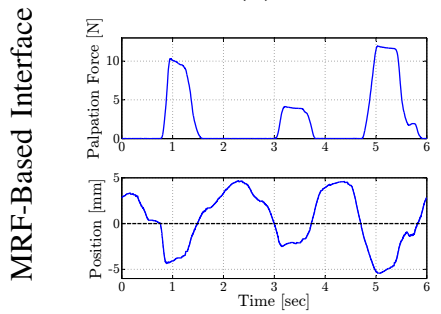

(c)

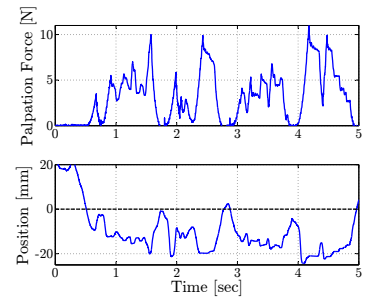

(d)

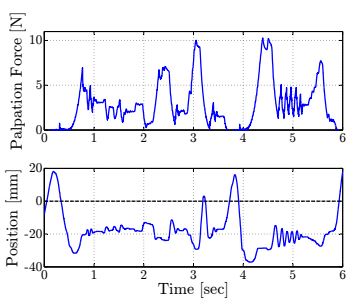

(e)

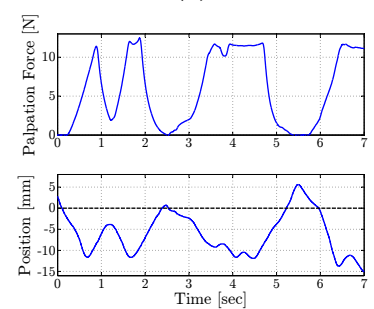

(f)
Fig. 11. Results of tissue palpation (Configuration $C$ ) on (a-c) an agar sample (10\% concentration) and (d-f) an ex vivo beef kidney. haptic devices was shown. Most commercial devices exhibit good transparency inside their region of stability. However, transparency will need to be sacrificed when the system tends to become unstable. Since this section only considers operation in stable mode, force feedback accuracy of the constructed device was studied on its own and no comparisons were made. Further statistical analysis and comparison between MRFbased haptic devices and conventional systems has been left for future work.

REMARK 4. In this section, the term "slave force/stiffness" is used to show the force/stiffness measured by the force sensor mounted at the based of the needle or palpator. In other words, this is the interaction force or the sensed stiffness during needle insertion or palpation, respectively. The term "desired torques" refers to the torques that are required to create the slave force or the sensed stiffness, based on the dynamics of the system. The term "estimated torque" or "estimated force/stiffness" corresponds to the estimated output torque of the clutch and the output forces of the haptic handle which are obtained by the ANN model, discussed in [2]. The term "feedback force/stiffness" refers to the force/stiffness felt by the force sensor attached to the haptic handle (i.e., the actual force as opposed to the estimated one).

REMARK 5. Transparency in a teleoperation system has two aspects; (i) how accurately the master creates the forces sensed on the slave side, and (ii) how accurately the slave follows the position of the master. Since the concentration of this work is on designing a suitable master device, the first aspect of transparency has only been considered in this section. Indeed, the second aspect is heavily dependent on the controller used for position tracking on the slave side.

1) Needle Insertion: To evaluate the performance of the haptic interface in providing accurate force feedback, first the needle insertion application was considered, and a few experiments were performed. The results are given in this section. These experiments were carried out on a variety of phantom and ex vivo samples using Configuration $B^{*}$. The torque control is performed at the clutch level, and consequently the joint torque and end effector force are controlled. As mentioned, in these experiments, the linear stage was used to command the needle insertion robot and guide the needle inside the sample. The results are shown in Fig. 12. The measured force from the slave side, the feedback force measured by the force sensor, and the estimated output force estimated by the ANN model is shown in this figure. As can be seen, the haptic interface can provide a fairly accurate force feedback on the master side. Three different concentrations of agar were used in the first three samples (Fig. 12 (a), (b), and (c) which have 3\%, 6\%, and $10 \%$ concentration rate respectively). As can be seen, the device provides accurate feedback to the user, which enables him/her to differentiate between the stiffnesses of the tissues. A gelatin sample (4\%; less stiff than the agar samples used previously) was also used for comparison (Fig. 12(d)). Next two combinations of gelatin and agar were used to simulate puncturing through different layers of tissue. The first one was a gelatin sample, which contained two spherical tumors made out of agar $(6 \%)$. The second sample consisted of layers 


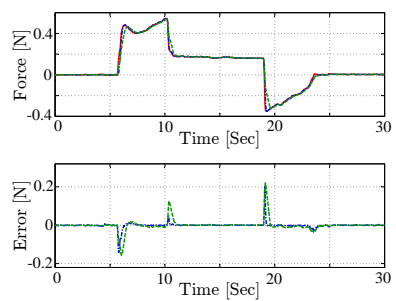

(a) Agar 3\%
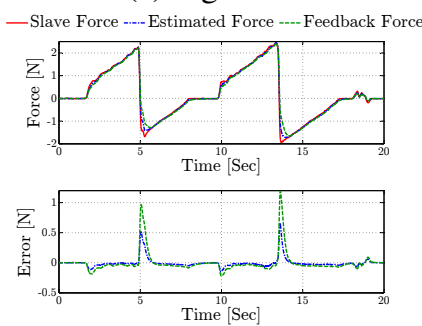

(c) Agar 10\%
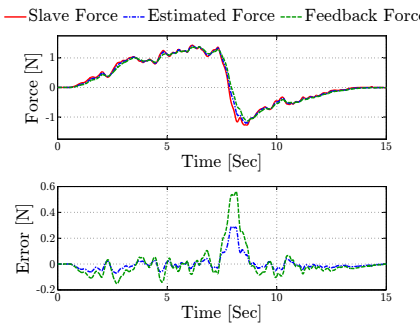

(e) Gelatin with Agar Tumors
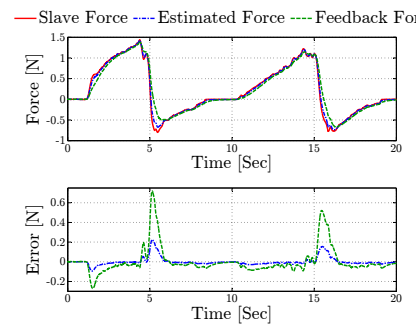

(b) Agar 6\%
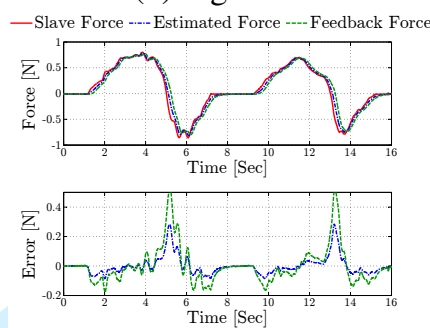

(d) Gelatin \%4
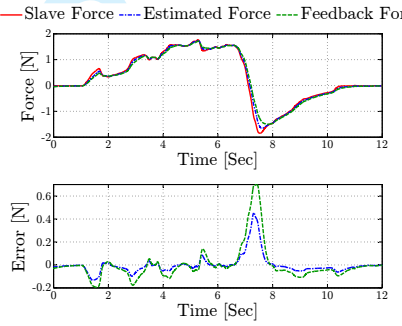

(f) Layered Sample

Fig. 12. Desired, estimated, and actual force feedback at the end effector during insertion and retraction of a needle (Configuration $B^{*}$ ) inside phantoms.

of gelatin (4\%) and agar (3\% and 6\%). The needle was inserted into the gelatin and was guided through these layers. Figs. 12(e,f) show the results. The ripples in the fedback force are caused during puncturing through these layers and as can be seen, the haptic device was able to recreate all these small deviations. The slow response of the torque feedback during sudden changes is mainly due to the tension propagation in the cables used for transmitting the torque from the clutches to the joint. Nevertheless, these results show the potential of the MRF-based haptic interface in providing accurate force feedback.

Next, ex vivo animal samples were used to test the accuracy of the device during needle insertion into animal tissue. For this purpose, several animal organs were used. Fig. 13 shows the results. Every tissue exhibits a different pattern of forces based on its stiffness, stickiness, and layers. In all cases, the MRF haptic device provided fairly accurate feedback, which enabled the differentiation between tissue properties.

2) Tissue Palpation: By taking advantage of Configuration $C^{*}$ for tissue palpation, further experiments were performed to assess the accuracy and capabilities of the device. In the results of this section, to evaluate the transparency of the system, the stiffness (impedance or ratio of force to displacement) of the sample was compared to the reflected stiffness of the haptic handle. First, palpation of phantom samples was performed. Three phantoms with different stiffnesses (i.e., gelatin sample (4\%), Agar samples (6\% and 10\%)) were used. Figs. 14(a-
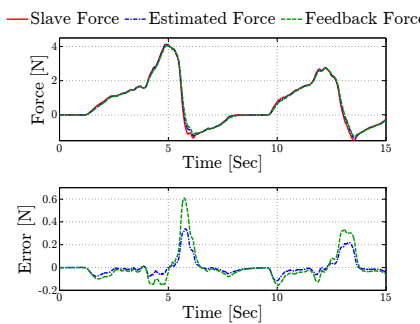

(a) Beef Heart
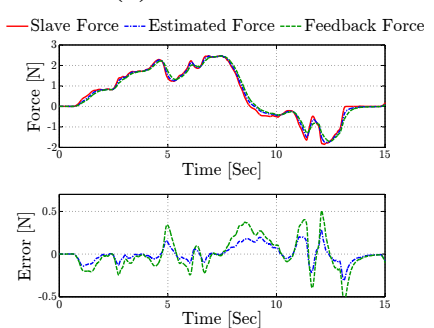

(c) Beef Kidney
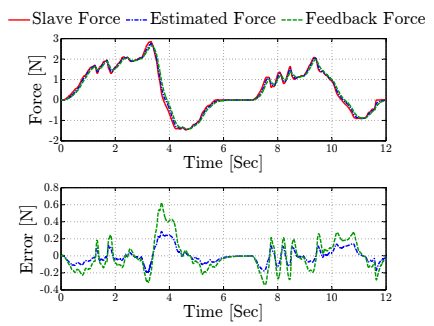

(e) Swine Lung

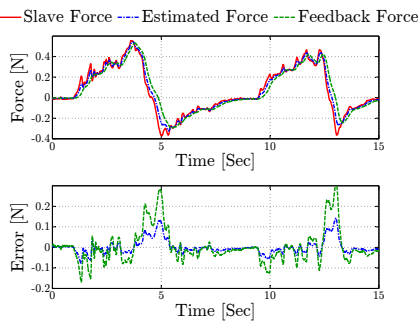

(b) Beef Liver
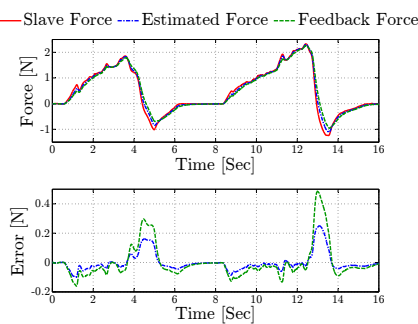

(d) Swine Heart

- Slave Force ----Estimated Force ---.-Feedback Force
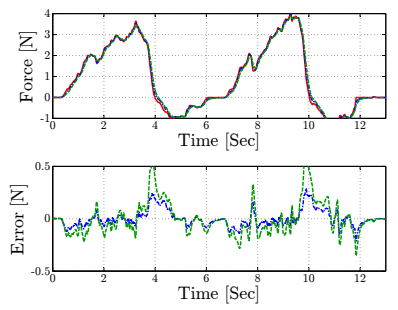

(f) Swine Stomach

Fig. 13. Desired, estimated, and actual feedback at the end effector during insertion and retraction of a needle (Configuration $B^{*}$ ) inside ex vivo samples.

c) show the results. It is evident that the haptic interface was capable of distinguishing between various stiffness values (environments).

Tumor detection is one of the main applications of palpation in medical interventions. For open procedures, the clinician can palpate using their hands but for robotics-assisted minimally invasive procedures, a haptic interface is needed [51]. To evaluate the performance of the haptic device in providing accurate and reliable force feedback for tumor detection during a minimally invasive intervention, a silicon model that contained two embedded silicon tumors (stiffer than the surrounding material) were used. These tumor-like samples were located at different depths - at $1 \mathrm{~cm}$ and $2 \mathrm{~cm}$. Three separate contacts were made with the sample, (i) several palpations of the surrounding silicon (no tumor) between 0s-12s; (ii) multiple contacts of the tissue which included the deep tumor 20s-30s; and (iii) final palpation of the tumor located at the depth of $1 \mathrm{~cm}$ in the period of 35s-50s. Fig 14 shows the results of the palpation. As can be seen, the MRF-based haptic device is capable of recreating the stiffness in all three cases, which enables the detection of the two tumors.

The performance of the haptic device in simulating stiffness of ex vivo animal organs was also studied. The results of the palpation experiments on several tissues are shown in Fig. 15. The outcomes in this section show the great potential of MRFbased actuators in haptic applications that require stable, high fidelity, and accurate force display. The cable-driven design of the system is one of its drawbacks due to the delay resulting 


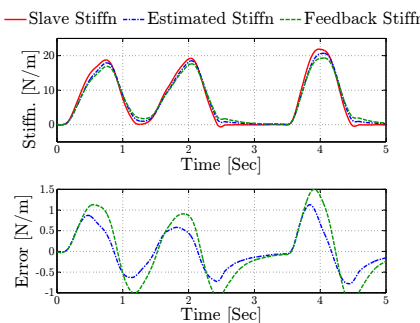

(a) Gelatin $4 \%$
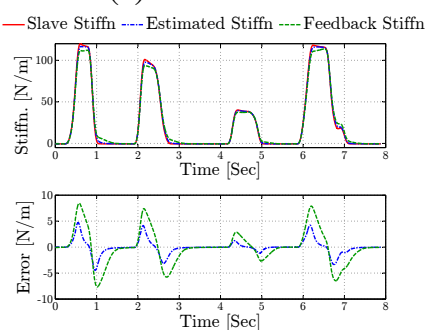

(c) Agar 10\%

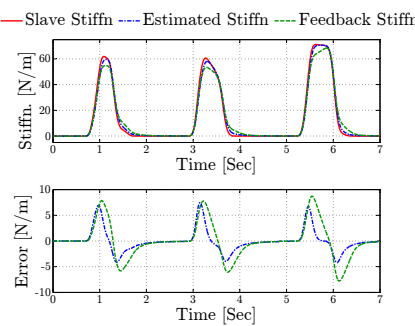

(b) Agar 6\%
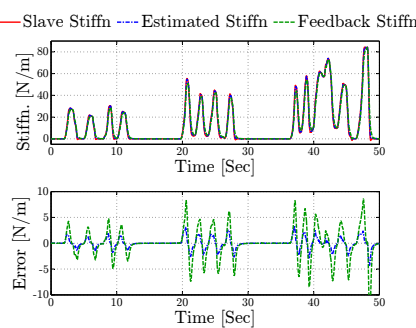

(d) Multi-Stiffness Silicon

Fig. 14. Desired, estimated, and actual stiffness at the end effector during palpation (Configuration $C^{*}$ ) of phantoms samples.

from the transmission of torque to the user's hand. This issue will be addressed in the next generation of the system.

\section{CONCLUDING REMARKS}

The design and evaluation of a prototype 2-DoF haptic interface based on MRF clutches were reported in this paper. The device was integrated and evaluated in a master-slave system for use in medical interventions consisting of needle insertion/steering, as well as, soft-tissue palpation. The stability and transparency of the system were rigorously tested using a wide range of phantom and ex vivo samples. In addition, the stability of the MRF-based haptic device was compared with that of two well-known off-the-shelf haptic interfaces.

The manufacturing cost of the clutch was one of our main design criteria. Given the simple mechanical design of the clutch, we believe that MRF-based clutches can be massproduced at a relatively low cost. The clutches presented in this paper were the first prototypes that were built inhouse at Western University's machine shop and used an Electric Discharge Machine (EDM) machining technique. The fabrication cost of each clutch was about $\mathrm{C} \$ 2000$, of which the labor cost was a major part (about $70 \%$ of the total). This cost can be reduced to a great extent through mass-production and using a cheaper means of machining.

The current design still has some shortcomings and drawbacks. Although the issue of heating did not cause significant problems at this stage, temperature sensing will be integrated in the next generation of the clutches to have a more accurate model of the system. The mass of the clutch should be reduced by decreasing the thickness of the outer casing through the use of more appropriate machining techniques. The issue of thickening is another problem in MRF-based systems. A new generation of commercial MRF consists of a stabilizer additive, which prolongs its efficient life. Nevertheless, in the long term, these systems will require maintenance due to this issue. Furthermore, the device was designed as a cabledriven system with the goal of making the haptic handle

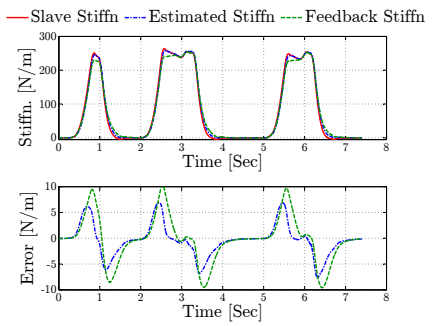

(a) Beef Heart

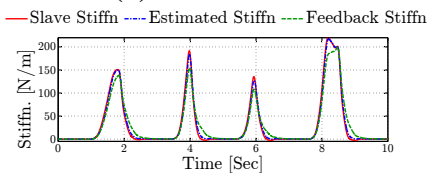

(c) Beef Kidney

- Slave Stiffn -...-Estimated Stiffn -.-.-Feedback Stiffn
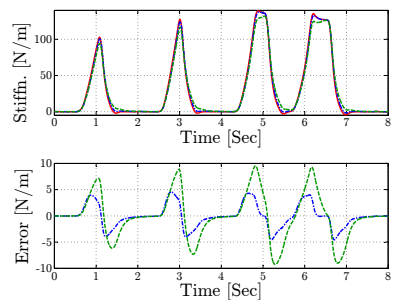

(e) Swine Lung

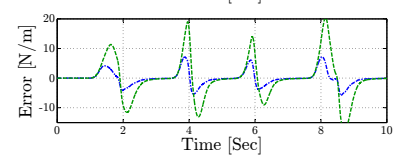

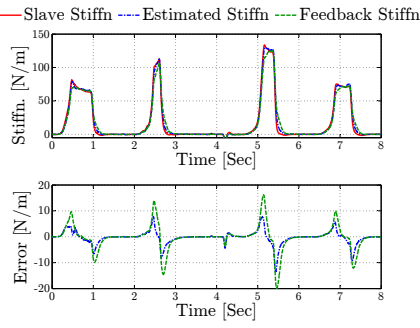

(b) Beef Liver

—-Slave Stiffn -..--Estimated Stiffn ----Feedback Stiff
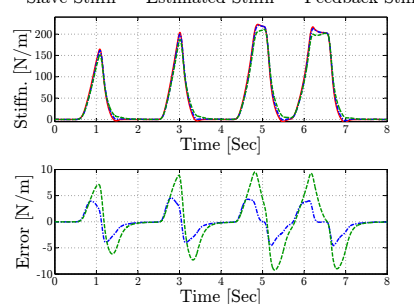

(d) Chicken Breast
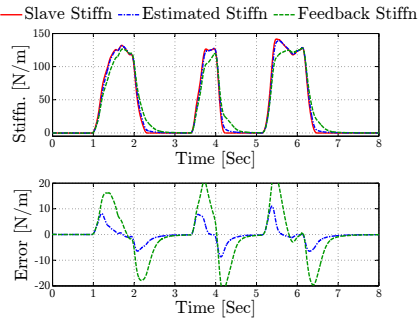

(f) Swine Stomach
Fig. 15. Desired, estimated, and actual stiffness at the end effector during palpation (Configuration $C^{*}$ ) of ex vivo organs.

simple and have low inertia. However, the delay caused by the propagation transmission via the cables reduced the bandwidth of the system. This mechanism will be replaced by a belt and pulley configuration in the future. In order to extend the haptic interface to 3-DoF, 2 more clutches are required. Based on our calculations, by reducing the weight of the next generation of the clutches, reconfiguring the location of the clutches in the device, and using a lightweight driving motor, a 3-DoF device can be made which has dimensions $200 \times 200 \times 100 \mathrm{~mm}$ and weighs $4 \mathrm{~kg}$. This shows great torque-to-size and weight ratios. Further investigation regarding stable performance of the haptic device will be carried out for teleoperation with time delays. Also, applications that involve larger environment forces, will be considered for further assessment of the system performance. In addition, the system will be tested in in vivo and active environments to study the effect of natural motions and forces (e.g., respiratory, cardiac, and body motions) on stability and effectiveness of the system.

While further investigations are required to study the effect of the device in specific medical applications, the results reported in this paper show the significant potential of MRF-based haptic devices for use in applications that require accurate and reliable performance.

\section{REFERENCES}

[1] N. Najmaei, P. Yadmellat, M. Kermani, and R. Patel, "Suitability of small-scale Magneto-Rheological fluid based clutches in haptic interfaces for improved performance," IEEE/ASME Transaction on Mechatronics, vol. in press., 2014. 
[2] N. Najmaei, A. Asadian, M. Kermani, and R. Patel, "MagnetoRheological actuators for haptic devices: Design, modeling, control, and validation of a prototype clutch," in IEEE International Conference on Robotics and Automation, May 2015.

[3] T. Coles, D. Meglan, and N. John, "The role of haptics in medical training simulators: A survey of the state of the art," IEEE Transactions on Haptics, vol. 4, pp. 51-66, Jan 2011.

[4] A. Okamura, C. Basdogan, S. Baillie, and W. Harwin, "Haptics in medicine and clinical skill acquisition [special section intro.]," Haptics, IEEE Transactions on, vol. 4, pp. 153-154, May 2011.

[5] R. Robison, C. Liu, and M. Apuzzo, "Man, mind, and machine: The past and future of virtual reality simulation in neurologic surgery," World Neurosurgery, vol. 76(5), pp. 1750-78, 2011.

[6] A. Hamed, S. Tang, and H. Ran, "Advances in haptics, tactile sensing, and manipulation for robot-assisted minimally invasive surgery, noninvasive surgery, and diagnosis," Journal of Robotics, vol. 12, pp. 1-14, 2012.

[7] K. Ohnishi, "Real world haptics and telehaptics for medical applications," in Industrial Electronics (ISIE), 2010 IEEE International Symposium on, pp. 11-14, July 2010.

[8] K. Kuchenbecker and G. Niemeyer, "Induced master motion in forcereflecting teleoperation," Journal of Dynamic Systems, Measurement, and Control, vol. 128, no. 4, pp. 800-810, 2006.

[9] R. Lozano, N. Chopra, and M. Spong, "Passivation of Force Reflecting Bilateral Teleoperation with Time Varying Delay," Journal of Mechatronics, vol. 12, pp. 215-223, 2002.

[10] I. Polushin, X. Liu, and C. Lung, "Stability of bilateral teleoperators with generalized projection-based force reflection algorithms," Automatica, vol. 48-6, pp. 1005-1016, 2012.

[11] A. Bicchi, M. Raugi, R. Rizzo, and N. Sgambelluri, "Analysis and design of an electromagnetic system for the characterization of MagnetoRheological fluids for haptic interfaces," IEEE Transactions on Magnetics, vol. 41, pp. 1876 - 1879, may 2005.

[12] A. Shafer and M. Kermani, "Design and validation of a MR clutch for practical control applications in human-friendly manipulation," in International Conference on Robotics and Automation, pp. 4266-71, 2011.

[13] M. Reed and W. Book, "Modeling and control of an improved dissipative passive haptic display," in IEEE International Conference on Robotics and Automation, vol. 1, pp. 311 - 318, Apr 2004.

[14] F. Ahmadkhanlou, G. Washington, and S. Bechtel, "Modeling and control of single and two degree of freedom Magneto-Rheological fluidbased haptic systems for telerobotic surgery," Journal of Intelligent Material Systems and Structures, vol. 20, pp. 1171-86, May 2009.

[15] J. An and D. Kwon, "Modeling of a Magneto-Rheological actuator including magnetic hysteresis," Journal of Intelligent Materials System Structures, vol. 14, pp. 541-550, 2003.

[16] N. Najmaei, P. Yadmellat, M. Kermani, and R. Patel, "Application of Magneto-Rheological fluid based clutches for improved performance in haptic interfaces," in IEEE International Conference on Robotics and Automation, June 2014.

[17] J. Melli-Huber, B. Weinberg, A. Fisch, J. Nikitczuk, C. Mavroidis, and C. Wampler, "Electro-Rheological fluidic actuators for haptic vehicular instrument controls," in Symposium on Haptic Interfaces for Virtual Environment and Teleoperator Systems, 2003.

[18] W. Li, B. Liu, P. Kosasih, and X. Zhang, "A 2-DOF MR actuator joystick for virtual reality applications," Sensors and Actuators, vol. 137, pp. 308-320, 2007.

[19] J. Furusho, M. Sakaguchi, N. Takesue, and K. Koyanagi, "Development of ER brake and its application to passive force display," Journal of Intelligent Material Systems and Structures, vol. 13, pp. 425-429, 2002.

[20] Y. Yamaguchi, S. Furusho, S. Kimura, and K. Koyanagi, "Development of high-perforemance MR actuator and its application to 2D force display," International Journal of Modern Physics B, vol. 19, pp. 14851491, 2005.

[21] D. Cassar and M. Saliba, "A force feedback glove based on MagnetoRheological fluid: Preliminary design issues," in IEEE Mediterranean Electrotechnical Conference, pp. 618-623, 26-28 2010.

[22] S. Winter and M. Bouzit, "Use of Magneto-Rheological fluid in a force feedback glove," IEEE Transactions on Neural Systems and Rehabilitation Engineering, vol. 15, pp. 2 -8, Mar 2007.

[23] Y. Nam, M. Park, and R. Yamane, "Smart Glove: Hand master using Magneto-Rheological fluid actuators," in Proceedings of SPIE, vol. 6794, pp. 679434-679434-6, 2007.

[24] J. Blake and H. Gurocak, "Haptic glove with MR brakes for virtual reality," IEEE/ASME Transactions on Mechatronics, vol. 14, pp. 606 -615 , oct. 2009.
[25] B. Liu, W. Li, P. Kosasih, and X. Zhang, "Development of an MRbrake-based haptic device," Smart Materials and Structures, vol. 15, pp. 1960-1966, 2006.

[26] P. Nguyen and B. Choi, "Selection of Magneto-Rheological brake types via optimal design considering maximum torque and constrained volume," Smart Materials and Structures, vol. 21-1, pp. 1-12, Dec 2012.

[27] A. Shafer and M. Kermani, "On the feasibility and suitability of MR fluid clutches in human-friendly manipulators," IEEE/ASME Transactions on Mechatronics, vol. 16-6, pp. 1073 - 82, Dec 2010.

[28] P. Yadmellat, A. Shafer, and M. Kermani, "Design and development of a safe robot manipulator using a new actuation concept," in International Conference on Robotics and Automation, pp. 337-42, 2013.

[29] A. Shafer and M. Kermani, MR Clutch with Sensors Measuring Electromagnetic Field Strength. US Patent 20130047772, Feb 2013.

[30] P. Yadmellat, A. Shafer, and M. Kermani, "Design and development of a single-motor, two-dof, safe manipulator," IEEE/ASME Transactions on Mechatronics, Accepted 2013.

[31] P. Yadmellat and M. Kermani, "Adaptive modeling of a MagnetoRheological clutch,” IEEE/ASME Transactions on Mechatronics, vol. 19, pp. 1716-1723, Oct 2014.

[32] D. Case, B. Taheri, and E. Richer, "Dynamical modeling and experimental study of a small-scale Magneto-Rheological damper," IEEE/ASME Transactions on Mechatronics, vol. 19, pp. 1015-1024, Jun 2014.

[33] W. Li, P. Yadmellat, and M. Kermani, "Linearized torque actuation using FPGA-controlled Magneto-Rheological actuators," IEEE/ASME Transactions on Mechatronics, vol. in press, pp. 1-9, May 2014.

[34] J. Colgate and G. Schenkel, "Passivity of a class of sampled-data systems: Application to haptic interfaces," Journal of Robotic Systems, vol. 14, pp. 37-47, 1997.

[35] M. Lin and M. Otaduy, Haptic Rendering: Foundations, Algorithms and Applications. A K Peters, 2008.

[36] H. Khalil, Nonlinear Systems. Prentice Hall, 2002.

[37] J. Colgate and J. Brown, "Factors affecting the Z-Width of a haptic display," in IEEE International Conference on Robotics and Automation, vol. 4, pp. 3205-10, 1999.

[38] E. Sontag, Nonlinear and Optimal Control Theory, ch. Input-to-state stability: Basic concepts and results, pp. 163-200. Springer-Verlag, 2006.

[39] S. Sandberg, "Some results on the theory of physical systems gov- erned by nonlinear functional equations," Journal of Bell System Technology, vol. 44, no. 1, pp. 871-98, 1965.

[40] I. Polushin, H. Marquez, A. Tayebi, and P. Liu, "A multichannel IOS small gain theorem for systems with multiple time-varying communication delays," IEEE Transactions on Automatic Control, vol. 54, pp. 404 -409 , Feb 2009.

[41] I. Polushin, A. Tayebi, and H. Marquez, "Control schemes for stable teleoperation with communication delay based on IOS small gain theorem," Automatica, vol. 42, no. 6, pp. 905-915, 2006.

[42] Geomatic Technologies Inc., Woburn MA, Specifications for the PHANTOM Desktop and PHANTOM Omni haptic devices, 2009.

[43] Quanser, Markham ON, 5-DOF Haptic Wand -Product Information Sheet S25, C ed., 2008.

[44] V. Hayward and O. Astley, "Performance measures for haptic interfaces," in Robotics Research (G. Giralt and G. Hirzinger, eds.), pp. 195-206, Springer London, 1996.

[45] H. Bassan, R. Patel, and M. Moallem, "A novel manipulator for percutaneous needle insertion: Design and experimentation," IEEE/ASME Transactions on Mechatronics, vol. 14(6), pp. 746-761, 2009.

[46] A. Asadian, Robotics-Assisted Needle Steering for Percutaneous Interventions: Modeling and Experiments. $\mathrm{PhD}$ thesis, University of Western Ontario, 2013.

[47] E. Chen, J. Novakofski, W. Jenkins, and J. O'Brien, W.D., "Young's modulus measurements of soft tissues with application to elasticity imaging," IEEE Transactions on Ultrasonics, Ferroelectrics, and Frequency Control, vol. 43, pp. 191-194, Jan 1996.

[48] A. Asadian, R. Patel, and M. Kermani, "Dynamics of translational friction in needletissue interaction during needle insertion," Annals of Biomedical Engineering, vol. 42(1), pp. 73-85, 2014.

[49] T. Hall, M. Bilgen, M. Insana, and T. Krouskop, "Phantom materials for elastography," IEEE Transactions on Ultrasonics, Ferroelectrics, and Frequency Control, vol. 44, pp. 1355-1365, Nov 1997.

[50] K. Ross and M. Scanlon, "Analysis of the elastic modulus of agar gel by indentation," Journal of Texture Studies, vol. 30, pp. 17-27, Nov 1999.

[51] A. Talasaz, R. Patel, and M. Naish, "Haptics-enabled teleoperation for robot-assisted tumor localization," in IEEE International Conference on Robotics and Automation, pp. 5340-5345, May 2010. 